Pacific Journal of Mathematics

THE BAIRE-CATEGORY METHOD IN SOME COMPACT 


\title{
THE BAIRE-CATEGORY METHOD IN SOME COMPACT EXTENSION PROBLEMS
}

\author{
Elżbieta Pol
}

\begin{abstract}
We characterize metrizable separable spaces $X$ such that almost every, in the sense of Baire category, embedding $h$ of $X$ into the Hilbert cube $I^{\omega}$ provides a compact extension $\overline{h(X)}$ such that the remainder $\overline{h(X)} \backslash h(X)$ has certain dimensional property (for instance, is $n$-dimensional, countable-dimensional or "metrically weakly infinitedimensional"). We obtain a characterization of metrizable separable spaces which have large transfinite dimension by means of compactifications. Two examples related to the results mentioned above are constructed.
\end{abstract}

1. Introduction. Consider the following two classes of separable metrizable spaces: the class $\left(P_{n}\right)$ of spaces $X$ with $\operatorname{dim} X \leq n$ and the class $\left(P_{\omega}\right)$ of countable-dimensional spaces (for terminology see $\S 2$ ). In this paper, for each of the classes $(P)$, we characterize the class of the spaces $X$ such that almost every, in sense of Baire category, homeomorphic embedding $h$ of $X$ into the Hilbert cube $I^{\omega}$ yields a compact extension $\overline{h(X)}$ of $h(X)$ whose remainder $\overline{h(X)} \backslash h(X)$ is in the class $(P)$.

In contrast with the classical result in dimension theory that if $X$ has a compact extension with $\operatorname{dim} \leq n$ (i.e. $\operatorname{dim} X \leq n$, by Hurewicz theorem) then almost every embedding $h: X \rightarrow I^{\omega}$ provides a compact extension $\overline{h(X)}$ with $\operatorname{dim} \overline{h(X)} \leq n$, the existence of a compactification $\tilde{Y}$ of $Y$ whose remainder $\tilde{Y} \backslash Y$ is in the class $(P)$, for any of the two $(P)$ we consider, is not enough to guarantee that almost every embedding $h$ : $Y \rightarrow I^{\omega}$ provides such a compactification $\overline{h(Y)}$ for $h(Y)$.

In the case of $\left(P_{n}\right)$ the characterization is simple: the class consists exactly of spaces which are unions of a compact set and an $n$-dimensional set $(\S 3)$.

To give a characterization for $\left(P_{\omega}\right)$, we introduce a somewhat weaker property than the weak infinite-dimensionality (in the sense of Smirnov). In particular, spaces having large transfinite dimension have this property, which yields a characterization of spaces with tr Ind, analogous to that given by Hurewicz for small transfinite dimension (\$4). 
We consider also (§5) spaces $X$ such that for almost every embedding $h: X \rightarrow I^{\omega}$ the remainder $\overline{h(X)} \backslash h(X)$ is "metrically weakly infinite-dimensional"; this class has a close connection with the class related to $\left(P_{\omega}\right)$.

We end the paper with two examples related to the subject of this paper (§6).

I would like to thank the referee for some valuable remarks.

2. Preliminaries. Our terminology follows [2], [3], and [4]. All spaces considered in this paper are assumed to be metrizable separable. If $A$ is a subset of $X$, then $B(A, \varepsilon)$ denotes an open $\varepsilon$-ball about $A$ with respect to a fixed metric in $X$. By the dimension we understand the covering dimension dim. A space $X$ is said to be $S$-weakly infinite-dimensional (weakly infinite-dimensional) if for every sequence $\left\{\left(A_{1}, B_{1}\right)\right.$, $\left.\left(A_{2}, B_{2}\right), \ldots\right\}$ of pairs of disjoint closed subsets of $X$ there exists a sequence $L_{1}, L_{2}, \ldots$ of closed subsets of $X$ such that $L_{i}$ is a partition between $A_{i}$ and $B_{i}$ (see [3], Definition 1.1.3), and $\bigcap_{i=1}^{N} L_{i}=\varnothing$ for some integer $N\left(\bigcap_{i=1}^{\infty} L_{i}=\varnothing\right)$. A space is countable-dimensional, if it is a union of countably many subspaces of dimension zero. The large (small) transfinite dimension tr Ind (trind) is the transfinite extension of the classical large (small) inductive dimension Ind (ind) (see [4], Definitions 1.1 and 1.2). A family $\left\{\left(A_{1}, B_{1}\right), \ldots,\left(A_{n}, B_{n}\right)\right\}$ of pairs of closed disjoint subsets of $X$ is essential, if for every sequence $L_{1}, \ldots, L_{n}$ of closed sets such that $L_{i}$ is a partition between $A_{i}$ and $B_{i}$ we have $\bigcap_{i=1}^{n} L_{i} \neq \varnothing$.

In the sequel we will often apply, without explicitly referring to, the following lemma on partitions (see [3], Lemma 1.2.9).

LemMa 2.1. Let $M$ be a subspace of a space $X$ and let $(A, B)$ be a pair of disjoint closed subsets of $X$. Then for every partition $L^{\prime}$ in the space $M$ between $M \cap \bar{U}$ and $M \cap \bar{V}$, where $U, V$ are open subsets of $X$ such that $A \subset U, B \subset V$ and $\bar{U} \cap \bar{V}=\varnothing$, there exists a partition $L$ in the space $X$ between $A$ and $B$ which satisfies the inclusion $M \cap L \subset L^{\prime}$.

By $I$ we denote the real interval $[-1,1], I^{\omega}$ denotes the Hilbert cube and let $\rho$ be a fixed metric in $I^{\omega}$. By $C\left(X, I^{\omega}\right)$ we denote the function space of all continuous mappings from $X$ into $I^{\omega}$ endowed with a complete metric

$$
d\left(f_{1}, f_{2}\right)=\sup \left\{\rho\left(f_{1}(x), f_{2}(x)\right): x \in X\right\} .
$$


The subspace of $C\left(X, I^{\omega}\right)$ consisting of all embeddings of $X$ into $I^{\omega}$ will be denoted by $E\left(X, I^{\omega}\right)$. A subset $A$ of a space $X$ is residual, if its complement $X \backslash A$ is a first category set (i.e. $X \backslash A$ is a union of countably many nowhere dense sets); a countable intersection of residual sets is residual in $X$, hence it is dense if $X$ is complete. We will need the following three classical theorems.

Theorem 2.2 (see [7], Ch. IV, §44, VI, Theorem 2). For any space $X$ the set $E\left(X, I^{\omega}\right)$ is a residual set in $C\left(X, I^{\omega}\right)$.

TheOREM 2.3 ( see [7], Ch. IV, \$45, VII, Theorem 4', cf. also [9]). If $A_{1}, \ldots, A_{k}$ are closed subsets of a space $X$ such that $A_{1} \cap \cdots \cap A_{k}=\varnothing$ then the set $\left\{h \in C\left(X, I^{\omega}\right): \overline{h\left(A_{1}\right)} \cap \cdots \cap \overline{h\left(A_{k}\right)}=\varnothing\right\}$ is a residual set in $C\left(X, I^{\omega}\right)$.

Theorem 2.4 (see [7], Ch. IV, §45, VII, Theorem 4'). If $A$ is a closed subset of a space $X$, then $\left\{h \in C\left(X, I^{\omega}\right): \operatorname{dim} \overline{h(A)} \leq \operatorname{dim} A\right\}$ is a residual set in $C\left(X, I^{\omega}\right)$.

3. Spaces whose almost every compact extension has remainder of $\operatorname{dim} \leq n$.

THEOREM 3.1. For any space $X$ and $n=0,1, \ldots$ the following conditions are equivalent:

(i) the set $\mathscr{H}=\left\{h \in E\left(X, I^{\omega}\right): \operatorname{dim}(\overline{h(X)} \backslash h(X)) \leq n\right\}$ is a residual set in $C\left(X, I^{\omega}\right)$ :

(ii) the set $\mathscr{H}$ is dense in $C\left(X, I^{\omega}\right)$;

(iii) there exists a compact set $K \subset X$ such that $\operatorname{dim}(X \backslash K) \leq n$.

Proof. The implication (i) $\Rightarrow$ (ii) is obvious. Let us show that (ii) $\Rightarrow$ (iii). First we will prove that if the condition (ii) holds then

(1) for every family $\left\{\left(A_{0}, B_{0}\right), \ldots,\left(A_{n}, B_{n}\right)\right\}$ of pairs of disjoint closed subsets of $X$ there exist closed sets $L_{0}, \ldots, L_{n}$ such that $L_{i}$ is a partition between $A_{i}$ and $B_{i}$ for $i=0, \ldots, n$ and the set $\bigcap_{i=0}^{n} L_{i}$ is compact.

Indeed, if $\left\{\left(A_{0}, B_{0}\right), \ldots,\left(A_{n}, B_{n}\right)\right\}$ is such a family then by Theorems 2.2 and 2.3 there exists $f \in E\left(X, I^{\omega}\right)$ such that $\overline{f\left(A_{i}\right)} \cap \overline{f\left(B_{i}\right)}=\varnothing$ for $i=0, \ldots, n$. Take $\varepsilon>0$ satisfying $\rho\left(\overline{f\left(A_{i}\right)}, \overline{f\left(B_{i}\right)}\right)>\varepsilon$. By (ii) there exists $h \in E\left(X, I^{\omega}\right)$ such that $d(f, h)<\varepsilon / 3$ and $\operatorname{dim}(\overline{h(X)} \backslash h(X)) \leq n$. We have then $\rho\left(h\left(A_{i}\right), h\left(B_{i}\right)\right) \geq \varepsilon / 3$ and thus $\overline{h\left(A_{i}\right)} \cap \overline{h\left(B_{i}\right)}=\varnothing$ for $i=$ $0, \ldots, n$. Let $U_{i}$ and $V_{i}$ be open subsets of $\overline{h(X)}$ containing $\overline{h\left(A_{i}\right)}$ and 
$\overline{h\left(B_{i}\right)}$ respectively with $\bar{U}_{i} \cap \bar{V}_{l}=\varnothing$. Since $\operatorname{dim}(\overline{h(X)} \backslash h(X)) \leq n$, then for $i=0, \ldots, n$ there exists a partition $L_{i}^{\prime}$ between $\bar{U}_{l} \cap(\overline{h(X)} \backslash h(X))$ and $\left.\bar{V}_{i} \cap \overline{(\overline{h(X)}} \backslash h(X)\right)$ in the space $\overline{h(X)} \backslash h(X)$ such that $\bigcap_{i=0}^{n} L_{i}^{\prime}=\varnothing$. By Lemma 2.1 for $i=0, \ldots, n$ there exists a partition $\tilde{L}_{i}$ between $\overline{h\left(A_{l}\right)}$ and $\overline{h\left(B_{i}\right)}$ in $\overline{h(X)}$ such that $\left.\tilde{L}_{i} \cap \overline{(\overline{h(X)}} \backslash h(X)\right) \subset L_{i}^{\prime}$. The set $\bigcap_{i=0}^{n} \tilde{L}_{l}$ is compact and it is contained in $h(X)$. Then the set $L_{i}=h^{-1}\left(\tilde{L}_{i} \cap h(X)\right)$ is a partition between $A_{i}$ and $B_{l}$ in $X$ such that the set $\bigcap_{i=0}^{n} L_{l}=$ $h^{-1}\left(\bigcap_{i=0}^{n} \tilde{L}_{i}\right)$ is compact, which finishes the proof of (1). To end the proof of (iii) put $K=X \backslash\{x \in X: x$ has a neighbourhood $U$ with $\operatorname{dim} U \leq n\}$ : we have $\operatorname{dim}(X \backslash K) \leq n$. If the set $K$ is not compact, then there exists a discrete sequence $\left\{x_{J}\right\}_{j=1}^{\infty}$ of points of $K$ and a discrete family $\left\{W_{J}\right\}_{J=1}^{\infty}$ of open subsets of $X$ such that $x_{j} \in W_{j}$. Since $x_{j} \in K$, then $\operatorname{dim} \bar{W}_{j}>n$ and thus there exists an essential family $\left\{\left(A_{0}^{j}, B_{0}^{J}\right), \ldots,\left(A_{n}^{j}, B_{n}^{j}\right)\right\}$ of pairs of closed disjoint subsets of $\bar{W}_{j}$. Then, for $i=0, \ldots, n$, the sets $A_{i}=\cup_{j=1}^{\infty} A_{i}^{J}$ and $B_{i}=\bigcup_{j=1}^{\infty} B_{i}^{J}$ are closed disjoint subsets of $X$ such that if $L_{i}$ is an arbitrary partition between $A_{i}$ and $B_{t}$, then the set $\bigcap_{t=0}^{n} L_{i}$ is not compact, since it intersects every set $\bar{W}_{\jmath}$. This is contrary to (1).

It remains to prove that (iii) $\Rightarrow$ (i). If $A_{l}=X \backslash B(K, 1 / i)$ for $i=1$, $2, \ldots$ then $\operatorname{dim} A_{l} \leq n$. By Theorems 2.2 and 2.4 the set $\mathscr{F}=\{h \in$ $E\left(X, I^{\omega}\right): \operatorname{dim} \overline{h\left(A_{i}\right)} \leq \operatorname{dim} A_{i}$ for $\left.i=1,2, \ldots\right\}$ is residual in $C\left(X, I^{\omega}\right)$. We shall show that $\operatorname{dim}(\overline{h(X)} \backslash h(X)) \leq n$ for every $h \in \mathscr{F}$. By the countable sum theorem it suffices to verify that $\overline{h(X)} \backslash h(X) \subset$ $\bigcup_{i=1}^{\infty} \overline{h\left(A_{i}\right)}$. This follows from the compactness of $K$ : if $x \in \overline{h(X)} \backslash h(X)$, then there exists a neighbourhood $U$ of $x$ in $\overline{h(X)}$ and a number $i$ such that $U \cap h(B(K, 1 / i))=\varnothing$; we have then $x \in \overline{h\left(A_{i}\right)}$.

REMARK 3.2. It is easy to see that the condition (1) described in the above proof is in fact equivalent to the conditions (i), (ii) and (iii) in Theorem 3.1. It is also easy to show by induction that $X$ satisfies (1) if and only if the strong inductive dimension which neglects the class of compact spaces of $X$ is not greater than $n$ (see [1] for the exact definition of this notion).

REMARK 3.3. Each of the conditions in Theorem 3.1 is equivalent to the following condition

(2) for each family $\left\{f_{l}\right\}_{i=0}^{n}$ of continuous functions of $X$ into the interval $I$ there exists $h \in E\left(X, I^{\omega}\right)$ such that $\operatorname{dim}(\overline{h(X)} \backslash h(X)) \leq n$, and for each $i=0, \ldots, n$ the function $f_{i} h^{-1}: h(X) \rightarrow I$ has a continuous extension $\tilde{f}_{i}: h(X) \rightarrow I$. 
The implication (i) $\Rightarrow$ (2) follows from Theorem 3.4 in [11] stating that for every function $f: X \rightarrow I$ the set $\left\{h \in E\left(X, I^{\omega}\right)\right.$ : the function $f h^{-1}$ has an extension $\tilde{f}: \overline{h(X)} \rightarrow I\}$ is residual in $C\left(X, I^{\omega}\right)$. Thus by Remark 3.2 it suffices to prove that $(2) \Rightarrow(1)$. Let $\left\{\left(A_{0}, B_{0}\right), \ldots,\left(A_{n}, B_{n}\right)\right\}$ be a sequence of pairs of disjoint closed subsets of $X$ and $f_{i}: X \rightarrow I$ be a function such that $f_{i}\left(A_{i}\right)=0$ and $f_{i}\left(B_{i}\right)=1$ for $i=0, \ldots, n$. By (2) there exists $h \in E\left(X, I^{\omega}\right)$ such that $\operatorname{dim}(\overline{h(X)} \backslash h(X)) \leq n$ and every function $f_{i} h^{-1}: h(X) \rightarrow I$ has an extension $\tilde{f}_{i}: \overline{h(X)} \rightarrow I$. For $i=0, \ldots, n$ we have $\tilde{f}_{i}\left(\overline{h\left(A_{i}\right)}\right)=0$ and $\tilde{f}_{i}\left(\overline{h\left(B_{i}\right)}\right)=1$, hence $\overline{h\left(A_{i}\right)} \cap \overline{h\left(B_{i}\right)}=\varnothing$. Now it is easy to construct the required partitions $L_{i}$ (compare the proof that (ii) $\Rightarrow(1)$ in Theorem 3.1).

4. Spaces whose almost every compact extension has countable-dimensional remainder.

THEOREM 4.1. For a space $X$ the following conditions are equivalent:

(i) the set $\mathscr{H}=\left\{h \in E\left(X, I^{\omega}\right): \overline{h(X)} \backslash h(X)\right.$ is countable-dimensional $\}$ is residual in $C\left(X, I^{\omega}\right)$ :

(ii) $X$ satisfies the following condition $(K): X$ contains a compact set $K$ such that $\operatorname{dim}(X \backslash U)<\infty$ for every open subset $U$ of $X$ which contains $K$;

(iii) the set $\mathscr{H}^{\prime}=\left\{h \in E\left(X, I^{\omega}\right): \overline{h(X)} \backslash h(X)\right.$ is locally finite-dimensional $\}$ is residual in $C\left(X, I^{\omega}\right)$.

Proof. Let us prove that (i) $\Rightarrow$ (ii). If the condition $(K)$ is not satisfied, then there exists a discrete family $\left\{U_{j}\right\}_{j=1}^{\infty}$ of open subsets of $X$ such that $\operatorname{dim} \bar{U}_{j} \geq j$ for $j=1,2, \ldots$, (the proof repeats the reasonings of Skljarenko [13]; cf. [2], Ch. X, §6, Lemmas 2 and 3). Let $\left\{\left(A_{i}^{j}, B_{i}^{j}\right)\right\}_{i=1}^{j}$ be an essential family in $\bar{U}_{j}$, then the sets $A_{i}=\bigcup_{j=i}^{\infty} A_{i}^{j}$ and $B_{i}=\bigcup_{j=i}^{\infty} B_{i}^{j}$ are disjoint closed subsets of $X$ for $i=1,2, \ldots$ By Theorems 2.2 and 2.3 the set $\mathscr{F}=\left\{h \in E\left(X, I^{\omega}\right): \overline{h\left(A_{i}\right)} \cap \overline{h\left(B_{i}\right)}=\varnothing\right.$ for $\left.i=1,2 \ldots\right\}$ is residual in $C\left(X, I^{\omega}\right)$. By (i) there exists $h \in \mathscr{F}$ such that $\overline{h(X)} \backslash h(X)=\bigcup_{i=1}^{\infty} Z_{i}$, where $\operatorname{dim} Z_{i} \leq 0$. By the separation theorem (see [3], Theorem 4.1.13) for each $i$ there exists a partition $L_{i}$ between the sets $\overline{h\left(A_{i}\right)}$ and $\overline{h\left(B_{i}\right)}$ such that $L_{i} \cap Z_{i}=\varnothing$. Then $L=\bigcap_{i=1}^{\infty} L_{i}$ is a compact subset of $\overline{h(X)}$ so that $h^{-1}(L)$ is a compact subset of $X$. Since the family $\left\{\bar{U}_{j}\right\}_{j=1}^{\infty}$ is discrete, $h^{-1}(L) \cap \bar{U}_{j}=\varnothing$ for $j \geq j_{0}$, where $j_{0}$ is some integer. Let $F$ $=\overline{\cup_{j=j_{0}}^{\infty} h\left(U_{j}\right)}$; since $L \cap F=\varnothing$, there exists an open subset $V$ of $\overline{h(X)}$ such that $L \subset V$ and $V \cap F=\varnothing$. By compactness of $L$ there exists $i_{0}$ such that $\bigcap_{i=1}^{i_{0}} L_{i} \subset V$ so that $\bigcap_{i=1}^{i_{0}} L_{i} \cap h\left(\bar{U}_{n}\right)=\varnothing$ for $n \geq j_{0}$. Take 
$n_{0}=\max \left(i_{0}, j_{0}\right)$. Putting $K_{i}=h^{-1}\left(L_{i}\right), i=1, \ldots, i_{0}$, we have

$$
\bigcap_{i=1}^{i_{0}}\left(K_{i} \cap \bar{U}_{n_{0}}\right)=\varnothing
$$

and $K_{i} \cap \bar{U}_{n_{0}}$ is a partition between $A_{i}^{n_{0}}$ and $B_{i}^{n_{0}}$, which is contrary to the assumption that the family $\left\{\left(A_{i}^{n_{0}}, B_{i}^{n_{0}}\right)\right\}_{i=1}^{n_{0}}$ is essential.

To show that (ii) $\Rightarrow$ (iii) put $F_{i}=X \backslash B(K, 1 / i)$ for $i=1,2, \ldots$; we have $\operatorname{dim} F_{i}<\infty$. By Theorems 2.2 and 2.4 the set $\mathscr{F}=\left\{h \in E\left(X, I^{\omega}\right)\right.$ : $\operatorname{dim} \overline{h\left(F_{i}\right)} \leq \operatorname{dim} F_{i}$ for $\left.i=1,2, \ldots\right\}$ is residual in $C\left(X, I^{\omega}\right)$. From the compactness of $K$ it follows easily that for each $h \in \mathscr{F}$ the set $\overline{h(X)} \backslash h(X)$ is locally finite-dimensional.

The implication (iii) $\Rightarrow$ (i) is obvious, since every locally finite-dimensional space is countable-dimensional.

REMARK 4.2. Let us notice that every $S$-weakly infinite-dimensional space (hence every space having large transfinite dimension $\operatorname{tr}$ Ind) satisfies the condition $(K)$. Indeed, let us recall that a space $X$ is $S$-weakly infinite-dimensional if and only if $S(X)=X \backslash \bigcup\{U: U$ is an open subspace of $X$ and $\operatorname{dim} U<\infty\}$ is a compact weakly infinite-dimensional subspace of $X$ such that for every open set $U \subset X$ which contains $S(X)$ we have $\operatorname{dim}(X \backslash U)<\infty$ (see [2], Ch. X, §6, Theorem 26).

By a theorem of Hurewicz (see [4], Theorem 4.15) a space $X$ has trind if and only if there exists $h \in E\left(X, I^{\omega}\right)$ such that $\overline{h(X)}$ is countable-dimensional. From Theorem 4.1 we obtain a corresponding characterization of spaces having tr Ind:

COROLlary 4.3. For a space $X$ the following conditions are equivalent:

(i) $X$ has large transfinite dimension $\mathrm{tr} I \mathrm{Ind}$;

(ii) the set $\mathscr{H}=\left\{h \in E\left(X, I^{\omega}\right): \overline{h(X)}\right.$ is countable-dimensional $\}$ is residual in $C\left(X, I^{\omega}\right)$.

Proof. The implication (i) $\Rightarrow$ (ii) has been proved by Luxemburg [8]. We shall prove that (ii) $\Rightarrow$ (i). If the set $\mathscr{H}$ is residual, then by Theorem 4.1 the space $X$ contains a compact set $K$ such that $\operatorname{dim}(X \backslash U)<\infty$ for every open subset of $X$ containing $K$. Since $X$ is homeomorphic to a subspace of a countable-dimensional space $\overline{h(X)}$, where $h$ is a member of $\mathscr{H}, X$ is countable-dimensional. It follows that $K$ is compact and countable-dimensional, hence $K$ has tr Ind, which implies that $X$ has tr Ind (see [4], Theorem 3.16). 
Let us note that other characterizations of metrizable separable spaces having tr Ind, by means of compactifications, were given in [4], Theorem 4.10 and [10], Theorem 10.1(a).

Corollary 4.4. For a space $X$ the following conditions are equivalent:

(i) $X$ is $S$-weakly infinite-dimensional;

(ii) the set $\mathscr{H}=\left\{h \in E\left(X, I^{\omega}\right): \overline{h(X)}\right.$ is weakly infinite-dimensional $\}$ is residual in $C\left(X, I^{\omega}\right)$.

Proof. If $X$ is $S$-weakly infinite-dimensional, then it satisfies $(K)$ and by Theorem 4.1 the set $\mathscr{H}=\left\{h \in E\left(X, I^{\omega}\right): \overline{h(X)} \backslash h(X)\right.$ is countable dimensional $\}$ is residual in $C\left(X, I^{\omega}\right)$. To prove (ii) it suffices to show that if $h(X)$ is $S$-weakly infinite-dimensional and $\overline{h(X)} \backslash h(X)=\cup_{i=1}^{\infty} Z_{i}$, where $\operatorname{dim} Z_{i} \leq 0$ for $i=1,2, \ldots$, then $\overline{h(X)}$ is weakly infinite-dimensional. Let $\left\{\left(A_{1}, B_{1}\right),\left(A_{2}, B_{2}\right), \ldots\right\}$ be a family of pairs of disjoint closed subsets of $\overline{h(X)}$. For $i=1,2, \ldots$ let $L_{2 i}$ be a partition between $A_{2 i}$ and $B_{2 i}$ in $\overline{h(X)}$ such that $L_{2 i} \cap Z_{i}=\varnothing$. Then the set $L=\bigcap_{i=1}^{\infty} L_{2 i}$ is a compact subset of $h(X)$, hence it is weakly infinite-dimensional. Thus for $i=1,2, \ldots$ there exists a partition $L_{2 i-1}^{\prime}$ between $A_{2 i-1} \cap L$ and $B_{2 i-1}$ $\cap L$ in $L$ such that $\bigcap_{i=1}^{\infty} L_{2 i-1}^{\prime}=\varnothing$. Taking partitions $L_{2 i-1}$ between $A_{2 i-1}$ and $B_{2 i-1}$ in $\overline{h(X)}$ such that $L_{2 i-1} \cap L \subset L_{2 i-1}^{\prime}$ we obtain a family $\left\{L_{i}\right\}_{i=1}^{\infty}$ of partitions such that $\bigcap_{i=1}^{\infty} L_{i}=\varnothing$.

It remains to prove that (ii) $\Rightarrow$ (i). If $\left\{\left(A_{1}, B_{1}\right),\left(A_{2}, B_{2}\right), \ldots\right\}$ is a family of pairs of disjoint closed subsets of $X$, then by (ii) and Theorems 2.2 and 2.3 there exists $h \in E\left(X, I^{\omega}\right)$ such that $\overline{h(X)}$ is weakly infinitedimensional, and $\overline{h\left(A_{i}\right)} \cap \overline{h\left(B_{i}\right)}=\varnothing$ for $i=1,2, \ldots$ If $L_{i}^{\prime}$ is a partition between $\overline{h\left(A_{i}\right)}$ and $\overline{h\left(B_{i}\right)}$ in $\overline{h(X)}$ such that $\bigcap_{i=1}^{N} L_{i}^{\prime}=\varnothing$, then $L_{i}=$ $h^{-1}\left(L_{i}^{\prime} \cap h(X)\right)$ is a partition between $A_{i}$ and $B_{i}$ in $X$ such that $\bigcap_{i=1}^{N} L_{i}$ $=\varnothing$.

\section{Spaces $S$-weakly infinite-dimensional modulo compacta.}

Definition 5.1. We say that a space $X$ is $S$-weakly infinite-dimensional modulo compacta (shortly, $S$-w.i.d. modulo compacta), if for every sequence $\left\{\left(A_{1}, B_{1}\right),\left(A_{2}, B_{2}\right), \ldots\right\}$ of pairs of closed disjoint subsets of $X$ there exists a sequence $\left\{L_{i}\right\}_{i=1}^{\infty}$ of closed subsets of $X$ such that $L_{i}$ is a partition between $A_{i}$ and $B_{i}$ and for some integer $N$ the set $\bigcap_{i=1}^{N} L_{i}$ is compact. 
Proposition 5.2. (i) Every space $X$ which is a union of a compact subspace and an $S$-weakly infinite-dimensional subspace is $S$-w.i.d. modulo compacta;

(ii) If a space $X$ is $S$-w.i.d. modulo compacta, then it has property $(K)$.

Proof. The proof of (i) is standard: if $X=K \cup Y$, where $K$ is compact, $Y$ is $S$-weakly infinite-dimensional, and $\left\{\left(A_{1}, B_{1}\right),\left(A_{2}, B_{2}\right), \ldots\right\}$ is a family of pairs of closed disjoint subsets of $X$, then we take open subsets $U_{i}$ and $V_{i}$ of $X$ such that $A_{i} \subset U_{i}, B_{i} \subset V_{i}$, and $\bar{U}_{i} \cap \bar{V}_{i}=\varnothing$. There exist partitions $L_{i}^{\prime}$ between $\bar{U}_{i} \cap Y$ and $\bar{V}_{i} \cap Y$ such that $\cap_{i=1}^{N} L_{i}^{\prime}=$ $\varnothing$ for some integer $N$. Take partitions $L_{i}$ between $A_{i}$ and $B_{i}$ such that $L_{i} \cap Y \subset L_{i}^{\prime}$; then the set $\bigcap_{i=1}^{N} L_{i} \subset K$ is compact.

The proof of (ii) follows easily from the fact that if the condition $(K)$ is not satisfied, then there exists a discrete family $\left\{U_{j}\right\}_{j=1}^{\infty}$ of open subsets of $X$ such that $\operatorname{dim} \bar{U}_{j} \geq j$ (compare the proof of the implication (i) $\Rightarrow$ (ii) in Theorem 4.1).

Definition 5.3. We say that a subspace $X \subset I^{\omega}$ is metrically $S$-weakly infinite-dimensional (shortly, $\mu$-S -w.i.d.) if for every sequence $\left\{\left(A_{1}, B_{1}\right),\left(A_{2}, B_{2}\right), \ldots\right\}$ of pairs of disjoint closed subsets of $X$ such that $\rho\left(A_{i}, B_{i}\right)>0$ there exists a partition $L_{i}$ between $A_{i}$ and $B_{i}, i=1,2, \ldots$, such that $\bigcap_{i=1}^{N} L_{i}=\varnothing$ for some integer $N$ (recall that $\rho$ is a fixed metric in $I^{\omega}$ ).

THEOREM 5.4. For a space $X$ the following conditions are equivalent:

(i) the set $\mathscr{H}=\left\{h \in E\left(X, I^{\omega}\right): \overline{h(X)} \backslash h(X)\right.$ is $\mu-S$-w.i.d. $\}$ is residual in $C\left(X, I^{\omega}\right)$;

(ii) $X$ is $S$-weakly infinite-dimensional modulo compacta.

Proof. To show that (i) $\Rightarrow$ (ii) let us take a sequence $\left\{\left(A_{1}, B_{1}\right)\right.$, $\left.\left(A_{2}, B_{2}\right), \ldots\right\}$ of pairs of disjoint closed subsets of $X$. By (i) and by Theorems 2.2 and 2.3 there exists $h \in E\left(X, I^{\omega}\right)$ such that $\overline{h\left(A_{i}\right)} \cap \overline{h\left(B_{i}\right)}$ $=\varnothing$, for $i=1,2, \ldots$, and the set $\overline{h(X)} \backslash h(X)$ is $\mu$-S-w.i.d. Take open sets $U_{i}$ and $V_{i}$ in $\overline{h(X)}$ containing $\overline{h\left(A_{i}\right)}$ and $\overline{h\left(B_{i}\right)}$ respectively such that $\bar{U}_{i} \cap \bar{V}_{i}=\varnothing$. Since $\rho\left(\bar{U}_{i}, \bar{V}_{i}\right)>0$, then there exist partitions $L_{i}^{\prime}$ between $\bar{U}_{i} \cap(\overline{h(X)} \backslash h(X))$ and $\bar{V}_{i} \cap(\overline{h(X)} \backslash h(X))$ such that $\bigcap_{i=1}^{N} L_{i}^{\prime}=\varnothing$ for some integer $N$. Finally, take partitions $\tilde{L}_{i}$ between $\overline{h\left(A_{i}\right)}$ and $\overline{h\left(B_{i}\right)}$ with

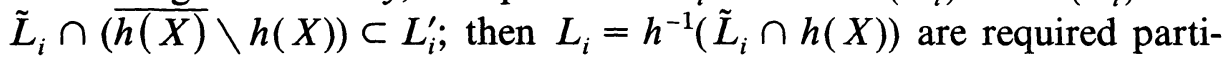
tions between $A_{i}$ and $B_{i}$. 
The first step in the proof that (ii) $\Rightarrow$ (i) is to show that if $\mathscr{A}=$ $\left\{\left(A_{1}, B_{1}\right), \ldots,\left(A_{N}, B_{N}\right)\right\}$ is a family of pairs of closed disjoint subsets of $X$ such that

(3) for $i=1, \ldots, N$ there exists a partition $L_{i}$ between $A_{i}$ and $B_{i}$ such that the $\operatorname{set} \bigcap_{i=1}^{N} L_{i}$ is compact, then

(4) the set $\mathscr{H}_{\mathscr{A}}=\left\{h \in E\left(X, I^{\omega}\right)\right.$ : for each $i=1, \ldots, N, \overline{h\left(A_{i}\right)} \cap \overline{h\left(B_{i}\right)}$ $=\varnothing$ and there exists a partition $K_{i}$ between $\overline{h\left(A_{i}\right)}$ and $\overline{h\left(B_{i}\right)}$ such that $\left.\bigcap_{i=1}^{N} K_{i} \backslash h(X)=\varnothing\right\}$ is residual in $C\left(X, I^{\omega}\right)$.

Indeed, for $i=1, \ldots, N$, let $E_{i}$ and $F_{i}$ be closed subsets of $X$ such that $L_{i}=E_{i} \cap F_{i}, A_{i} \subset E_{i}, B_{i} \subset F_{i}, A_{i} \cap F_{i}=\varnothing, B_{i} \cap E_{i}=\varnothing$ and $E_{i} \cup F_{i}$ $=X$. Then the set $C=\bigcap_{i=1}^{N} L_{i}=\bigcap_{i=1}^{N} E_{i} \cap \bigcap_{i=1}^{N} F_{i}$ is a compact subset of $X$. Let $V_{k}=B(C, 1 / k)$ for $k=1,2, \ldots$, ; we have $\left(\cap_{i=1}^{N} E_{i} \cap \cap_{i=1}^{N} F_{i}\right) \backslash V_{k}$ $=\varnothing$. Hence for each $k$ and a family $\left\{E_{i} \backslash V_{k}: i=1, \ldots, N\right\} \cup\left\{F_{i} \backslash V_{k}\right.$ : $i=1, \ldots, N\}$ of closed subsets of $X$ we have $\bigcap_{i=1}^{N}\left(E_{i} \backslash V_{k}\right) \cap\left(F_{i} \backslash V_{i}\right)=$ $\varnothing$. By Theorems 2.2 and 2.3 the sets $\mathscr{H}_{1}=\left\{h \in E\left(X, I^{\omega}\right)\right.$ : $\cap_{i=1}^{N} \overline{h\left(E_{i} \backslash V_{k}\right)} \cap \bigcap_{i=1}^{N} \overline{h\left(F_{i} \backslash V_{i}\right)}=\varnothing$ for $\left.k=1,2 \ldots\right\}$ and $\mathscr{H}_{2}=\{h$ $\in E\left(X, I^{\omega}\right): \overline{h\left(A_{i}\right)} \cap \overline{h\left(F_{i}\right)}=\varnothing$ and $\overline{h\left(E_{i}\right)} \cap \overline{h\left(B_{i}\right)}=\varnothing$ for $i=1$, $2 \ldots\}$ are residual in $C\left(X, I^{\omega}\right)$. We will show that $\mathscr{H}_{\mathscr{A}} \supseteq \mathscr{H}_{1} \cap \mathscr{H}_{2}$. For $i=1, \ldots, N$ let $K_{i}=\overline{h\left(E_{i}\right)} \cap \overline{h\left(F_{i}\right)}$; since $h \in \mathscr{H}_{2}$, then the set $K_{i}$ is a partition between $\overline{h\left(A_{i}\right)}$ and $\overline{h\left(B_{i}\right)}$ in $\overline{h(X)}$. Let us verify that

$$
\bigcap_{i=1}^{N} K_{i} \backslash h(X)=\varnothing .
$$

Suppose, to the contrary, that there exists a point $x \in\left(\bigcap_{i=1}^{N} \overline{h\left(E_{i}\right)} \cap\right.$ $\left.\bigcap_{i=1}^{N} \overline{h\left(F_{i}\right)}\right) \backslash h(X)$. Since $\{x\}$ and $h(C)$ are disjoint compact subsets of $\overline{h(X)}$, there exists a neighbourhood $V$ of the point $x$ in $\overline{h(X)}$ such that $\bar{V} \cap h(C)=\varnothing$. Take an integer $k$ such that $V_{k} \cap h^{-1}(V)=\varnothing$. For each $i$ we have

$$
x \in \overline{h\left(E_{i}\right) \cap V} \cap \overline{h\left(F_{i}\right) \cap V} \subset \overline{h\left(E_{i} \backslash V_{k}\right)} \cap \overline{h\left(F_{i} \backslash V_{k}\right)}
$$

which is a contradiction, since $h \in \mathscr{H}_{1}$. This ends the proof of (4).

Now suppose that $X$ is $S$-w.i.d. modulo compacta. Let $\mathscr{D}=$ $\left\{\left(C_{1}, D_{1}\right),\left(C_{2}, D_{2}\right), \ldots\right\}$ be a family of pairs of disjoint closed subsets of $I^{\omega}$ such that for every pair $(A, B)$ of disjoint closed subsets of $I^{\omega}$ there exists an $i$ such that $A \subset C_{i}$ and $B \subset D_{i}$. Let $\mathscr{U}_{1}$ be a maximal family of 
disjoint open subsets of $C\left(X, I^{\omega}\right)$ of diameter $<1$. If $\mathscr{U}_{i-1}$ is defined, then for each $U \in \mathscr{U}_{i-1}$ let $\mathscr{U}_{i, U}$ be a maximal family of disjoint open subsets of $U$ of diameter $<1 / i$ and $\mathscr{U}_{i}=\bigcup_{U \in \mathscr{U}_{i-1}} \mathscr{U}_{i, U}$.

Let us fix an integer $i$. For each $U \in \mathscr{U}_{i}$ let us choose $f_{U} \in U$ and consider the family

$$
\mathscr{D}_{U}=\left\{\left(f_{U}^{-1}\left(C_{1}\right), f_{U}^{-1}\left(D_{1}\right)\right),\left(f_{U}^{-1}\left(C_{2}\right), f_{U}^{-1}\left(D_{2}\right)\right), \ldots\right\}
$$

of pairs of disjoint closed subsets of $X$. Let $\mathscr{K}(U)$ be the family of all finite subsequences $\mathscr{A}=\left\{\left(A_{1}, B_{1}\right), \ldots,\left(A_{N}, B_{N}\right)\right\}$ of the family $\mathscr{D}_{U}$ for which the condition (3) is satisfied. By (4), the set $\mathscr{H}_{\mathscr{A}}$ is residual in $C\left(X, I^{\omega}\right)$ for every $\mathscr{A} \in \mathscr{K}(U)$. Since $|\mathscr{K}(U)| \leq \chi_{0}$, then the set $\mathscr{H}(U)$ $=\bigcap_{\mathscr{A} \in \mathscr{K}(U)} \mathscr{H}_{\mathscr{A}}$ is also residual in $C\left(X, I^{\omega}\right)$. Since the family $\mathscr{U}_{i}$ consists of disjoint and open subsets of $C\left(X, I^{\omega}\right)$ and $\overline{U \mathscr{U}_{i}}=C\left(X, I^{\omega}\right)$, then the set

$$
\mathscr{H}_{i}=\bigcup_{U \in \mathscr{U}_{i}}(\mathscr{H}(U) \cap U)
$$

is residual in $C\left(X, I^{\omega}\right)$. Finally, the set $\mathscr{H}^{\prime}=\bigcap_{i=1}^{\infty} \mathscr{H}_{i}$ is residual in $C\left(X, I^{\omega}\right)$.

It remains to prove that $\mathscr{H}^{\prime} \subset \mathscr{H}$, i.e. that for every $h \in \mathscr{H}^{\prime}$ the set $\overline{h(X)} \backslash h(X)$ is $\mu$-S-w.i.d. Let $\left\{\left(A_{1}, B_{1}\right),\left(A_{2}, B_{2}\right), \ldots\right\}$ be a family of disjoint closed subsets of $\overline{h(X)} \backslash h(X)$ such that $\rho\left(A_{i}, B_{i}\right)>0$ for $i=1$, $2, \ldots$. Then for each $i$ the closures $\bar{A}_{i}$ and $\bar{B}_{i}$ of $A_{i}$ and $B_{i}$ in $I^{\omega}$ are disjoint and thus there exists an integer $j(i)$ and a real number $\varepsilon_{i}>0$ such that $B\left(A_{i}, \varepsilon_{i}\right) \subset C_{j(i)}$ and $B\left(B_{i}, \varepsilon_{i}\right) \subset D_{j(i)}$. For each $i$ take $\eta_{i}>0$ such that the sets $C_{i}^{*}=B\left(C_{j(i)}, \eta_{i}\right)$ and $D_{i}^{*}=\overline{B\left(D_{j(i)}, \eta_{i}\right)}$ are disjoint. Consider the family $\left\{\left(h^{-1}\left(C_{1}^{*}\right), h^{-1}\left(D_{1}^{*}\right)\right),\left(h^{-1}\left(C_{2}^{*}\right), h^{-1}\left(D_{2}^{*}\right)\right), \ldots\right\}$. Since $X$ is $S$-w.i.d. modulo compacta, there exists an integer $N$ and partitions $L_{i}$ between $h^{-1}\left(C_{i}^{*}\right)$ and $h^{-1}\left(D_{i}^{*}\right)$, for $i=1, \ldots, N$, such that the set $\bigcap_{i=1}^{N} L_{i}$ is compact. Let $\varepsilon=\min \left\{\varepsilon_{1}, \ldots, \varepsilon_{N}, \eta_{1}, \ldots, \eta_{N}\right\}$ and let $m$ be a natural number such that $1 / m<\epsilon$. There exists exactly one $U \in \mathscr{U}_{m}$ such that $h \in U$. Observe that $f_{U}^{-1}\left(C_{j(i)}\right) \subset h^{-1}\left(C_{1}^{*}\right)$ and $f_{U}^{-1}\left(D_{j(i)}\right) \subset h^{-1}\left(D_{i}^{*}\right)$. Indeed, if $x \in f_{U}^{-1}\left(C_{j(i)}\right)$, then since $d\left(h, f_{U}\right)<1 / m<\varepsilon$ we have $\rho\left(h(x), f_{U}(x)\right)<\varepsilon$ and thus $h(x) \in B\left(C_{j(i)}, \varepsilon\right) \subset B\left(C_{j(i)}, \eta_{i}\right) \subset C_{i}^{*}$ i.e. $x \in h^{-1}\left(C_{i}^{*}\right)$.

Thus, for $i=1, \ldots, N$, the set $L_{i}$ is a partition between the sets $f_{U}^{-1}\left(C_{j(i)}\right)$ and $f_{U}^{-1}\left(D_{j(i)}\right)$ and the set $\bigcap_{i=1}^{N} L_{i}$ is compact. Hence the family

$$
\mathscr{A}=\left\{\left(f_{U}^{-1}\left(C_{j(1)}\right), f_{U}^{-1}\left(D_{j(1)}\right)\right), \ldots,\left(f_{U}^{-1}\left(C_{j(N)}\right), f_{U}^{-1}\left(D_{j(N)}\right)\right)\right\} \in \mathscr{K}(U) .
$$


Since $h \in \mathscr{H}(U)$, then $h \in \mathscr{H}_{\mathscr{A}}$ and thus there exist partitions $K_{i}$ between $\overline{h\left(f_{U}^{-1}\left(C_{j(i)}\right)\right)}$ and $\overline{h\left(f_{U}^{-1}\left(D_{j(i)}\right)\right)}$ such that $\bigcap_{i=1}^{N} K_{i} \backslash h(X)=\varnothing$. Observe that $A_{i} \subset h f_{U}^{-1}\left(C_{j(i)}\right)$ and $B_{i} \subset h f_{U}^{-1}\left(D_{j(i)}\right)$. Indeed, we shall show that $h^{-1}\left(A_{i}\right) \subset f_{U}^{-1}\left(C_{j(i)}\right)$ and $h^{-1}\left(B_{i}\right) \subset f_{U}^{-1}\left(D_{j(i)}\right)$. Let $a \in h^{-1}\left(A_{i}\right)$; then $\rho\left(f_{U}(a), h(a)\right)<\varepsilon \leq \varepsilon_{i}$ and thus $f_{U}(a) \in B\left(A_{i}, \varepsilon_{i}\right) \subset C_{j(i)}$, i.e. $a \in$ $f_{U}^{-1}\left(C_{j(i)}\right)$. It follows that $\left.K_{i}^{\prime}=K_{i} \cap \overline{(h(X)} \backslash h(X)\right)$ is a partition between $A_{i}$ and $B_{i}$ in $\overline{h(X)} \backslash h(X)$ for $i=1, \ldots, N$ and $\bigcap_{i=1}^{N} K_{i}^{\prime}=\varnothing$, which ends the proof.

6. Examples and remarks.

EXAmPLE 6.1. A space $X$ satisfying the condition $(K)$ which is not $S$-weakly infinite-dimensional modulo compacta.

Let $Z=\left\{0,1, \frac{1}{2}, \frac{1}{3}, \ldots\right\}$ be a subspace of the real line and let $E_{n}=$ $\left\{\left\{x_{j}\right\} \in I^{\omega}:-1<x_{j}<1\right.$ for $j=1,2, \ldots, n$ and $x_{j}=0$ for $j=n+1$, $n+2, \ldots$,$\} , for n=1,2, \ldots$ Let

$$
X=\left(I^{\omega} \times\{0\}\right) \cup \bigcup_{n=1}^{\infty}\left(E_{n} \times\left\{\frac{1}{n}\right\}\right)
$$

be a subspace of the Cartesian product $I^{\omega} \times Z$. Put $A_{i}=\left\{\left(\left\{x_{j}\right\}, 0\right) \in I^{\omega}\right.$ $\left.\times\{0\}: x_{i}=-1\right\}$ and $B_{i}=\left\{\left(\left\{x_{j}\right\}, 0\right) \in I^{\omega} \times\{0\}: x_{i}=1\right\} ; A_{i}$ and $B_{i}$ are compact disjoint subsets of the space $X$. Let $L_{i}$ be an arbitrary partition between $A_{i}$ and $B_{i}$. We shall show that for every integer $N$ the set $\bigcap_{i=1}^{N} L_{i}$ is not compact (this implies that $X$ is not $S$-w.i.d. modulo compacta; some other properties of $X$ follow from Remark 6.3 below- see Corollary below). Let $I_{n}=\left\{\left\{x_{j}\right\} \in I^{\omega}:-1 \leq x_{j} \leq 1\right.$ for $j=1, \ldots, n$ and $x_{j}=0$ for $\left.j=n+1, n+2, \ldots\right\}$. Put

$$
X^{\prime}=\left(I^{\omega} \times\{0\}\right) \cup \bigcup_{n=1}^{\infty}\left(I_{n} \times\left\{\frac{1}{n}\right\}\right) \subset I^{\omega} \times Z .
$$

For each $i$ take a partition $L_{i}^{\prime}$ between $A_{i}$ and $B_{i}$ in $X^{\prime}$ such that $L_{i}^{\prime} \cap X \subset L_{i}$. For every $i$ there exists integer $n_{i} \geq 1$ such that for any $n \geq n_{i}$ the set $L_{i}^{\prime} \cap\left(I_{n} \times\{1 / n\}\right)$ is a partition between the sets $\left\{\left(\left\{x_{j}\right\}, 1 / n\right):\left\{x_{j}\right\} \in I_{n}, x_{i}=-1\right\}$ and $\left\{\left(\left\{x_{j}\right\}, 1 / n\right):\left\{x_{j}\right\} \in I_{n}, x_{i}=1\right\}$ in the space $I_{n} \times\{1 / n\}$. Let $N$ be an arbitrary integer and let $n \geq$ $\max \left\{n_{1}, \ldots, n_{N}, N+1\right\}$. Then for $i=1, \ldots, N$ the set $L_{i}^{\prime} \cap\left(I_{N+1} \times\right.$ $\{1 / n\})$ is a partition between the opposite faces $\left\{\left(\left\{x_{j}\right\}, 1 / n\right) \in I_{N+1} \times\right.$ $\left.\{1 / n\}: x_{i}=-1\right\}$ and $\left\{\left(\left\{x_{j}\right\}, 1 / n\right) \in I_{N+1} \times\{1 / n\}: x_{i}=1\right\}$ of the cube $I_{N+1} \times\{1 / n\}$. Hence the set $\bigcap_{i=1}^{N} L_{i}^{\prime} \cap\left(I_{N+1} \times\{1 / n\}\right)$ contains a continuum $C$ meeting the faces $\left\{\left(\left\{x_{j}\right\}, 1 / n\right) \in I_{N+1} \times\{1 / n\}: x_{N+1}=-1\right\}$ and $\left\{\left(\left\{x_{j}\right\}, 1 / n\right) \in I_{N+1} \times\{1 / n\}: x_{N+1}=1\right\}$ (see [12], Lemma 5.2). For 
$m=1,2 \ldots$ take

$$
a_{m} \in \bigcap_{i=1}^{N} L_{i}^{\prime} \cap\left\{\left(\left\{x_{j}\right\}, \frac{1}{n}\right) \in I_{N+1} \times\left\{\frac{1}{n}\right\}: x_{N+1}=1-\frac{1}{m}\right\} .
$$

Then the sequence $\left\{a_{m}\right\}_{m=1}^{\infty}$ is contained in $\bigcap_{i=1}^{N} L_{i}$ and has no accumulation point in $X$. Thus the set $\bigcap_{i=1}^{N} L_{i}$ is not compact.

EXAmple 6.2. A space $X$ such that $X$ has tr Ind and yet $X$ has no compactification with $S$-weakly infinite-dimensional remainder.

Before we proceed to the construction let us recall that the deficiency of a space $X$, def $X$, is defined as $\min \{\operatorname{dim}(\tilde{X} \backslash X): \tilde{X}$ is a compactification of $X\}$ (see [5]).

Let $X=\oplus_{n=1}^{\infty} X_{n} \cup\{p\}$, where $X_{n}$ is a separable space such that $\operatorname{def} X_{n} \geq n$ and the base of neighbourhoods of $p$ consists of the sets of the form $\bigcup_{n=k}^{\infty} X_{n}$, where $k=1,2, \ldots$ For example, we can take $X_{n}$ $=I^{n+1} \backslash\left\{\left(x_{1}, \ldots, x_{n}\right): x_{n+1}=1\right.$ and $-1<x_{i}<1$ for $\left.i=1, \ldots, n\right\}$ (see [6], Ch. VI, Research problem D); notice that inequality $\operatorname{def} X_{n} \geq n$ follows from the fact that if $L_{i}$ is a partition between the compact sets $A_{l}=\left\{\left(x_{i}, \ldots, x_{n+1}\right) \in I^{n+1}: x_{i}=-1\right\}$ and $B_{i}=\left\{\left(x_{1}, \ldots, x_{n+1}\right)\right.$ $\left.\in I^{n+1}: x_{i}=1\right\}$ in $X_{n}$, for $i=1, \ldots, n$, then the set $\bigcap_{i=1}^{n} L_{i}$ is not compact (compare Remark 6.3 below). Suppose that the space $X$ has a compactification $\tilde{X}$ such that the remainder $\tilde{X} \backslash X$ is $S$-weakly infinite-dimensional. Then $\tilde{X} \backslash X$ contains a compact set $K$ such that $\operatorname{dim}(\tilde{X} \backslash X \backslash W)<\infty$ for every open set $W$ in $\tilde{X} \backslash X$ containing $K$. Let $U$ and $V$ be neighbourhoods of the set $K$ and the point $x$ respectively in the space $\tilde{X}$ such that $\bar{U} \cap \bar{V}=\varnothing$. Then the set $(\tilde{X} \backslash X) \backslash U$ has a finite dimension, hence the set $V \subset X \cup(\tilde{X} \backslash X) \backslash U$ has $\operatorname{def} V<\infty$. This is a contradiction, since $V$ contains almost all $X_{n}$ 's and def $X_{n} \geq n$.

REMARK 6.3. In the sequel by a compactness degree of a space $X$ ( $\mathrm{cmp} X$ ) we understand the transfinite invariant defined as follows: $\mathrm{cmp} X$ $=-1$ if $X$ is compact and $\operatorname{cmp} X \leq \alpha$ if $X$ has a base $\mathscr{B}$ of open sets such that cmp Fr $U<\alpha$ for every $U \in \mathscr{B}$ (see [5] and [14]).

Proposition. Suppose that a space $X$ satisfies one of the following conditions:

(i) there is an embedding $h: X \rightarrow I^{\omega}$ such that the set $\overline{h(X)} \backslash h(X)$ is $\mu$-S-weakly infinite-dimensional,

(ii) the transfinite compactness degree $\mathrm{cmp} X$ is defined. 
(5) for every sequence $\left\{\left(A_{1}, B_{1}\right),\left(A_{2}, B_{2}\right), \ldots\right\}$ of pairs of disjoint compact subsets of $X$ there exist partitions $L_{i}$ between $A_{i}$ and $B_{i}$ such that for some integer $N$ the set $\bigcap_{i=1}^{N} L_{i}$ is compact.

Proof. In the case when $X$ satisfies (i) the proof is similar to the proof of the implication (i) $\Rightarrow$ (ii) in Theorem 5.4. Now suppose that $\mathrm{cmp} X$ is defined. We apply the transfinite induction with respect to $\alpha=\operatorname{cmp} X$. If $\alpha=-1$, then $X$ is compact and the condition (5) is obviously satisfied. Now assume that the proposition is proved if $\operatorname{cmp} X<\alpha$ and let $X$ be a space such that $\operatorname{cmp} X \leq \alpha$. Let $\left\{\left(A_{1}, B_{1}\right),\left(A_{2}, B_{2}\right), \ldots\right\}$ be a sequence of pairs of disjoint compact subsets of $X$. Since $A_{i}$ is compact and cmp $X \leq \alpha$, there exist open subsets $U_{1}, \ldots, U_{k}$ of $X$ such that $A_{1} \subset \bigcup_{i=1}^{k} U_{i}$ and for each $i=1, \ldots, k$ we have $\bar{U}_{i} \cap B_{1}=\varnothing$ and $\operatorname{cmpFr} U_{i} \leq \alpha^{\prime}$ for some $\alpha^{\prime}<\alpha$. By the inductive assumption each Fr $U_{i}$ satisfies (5), hence the set $\bigcup_{i=1}^{k} \operatorname{Fr} U_{i}$ satisfies (5) since the property (5) is finitely additive. It is easy to see that the set $L_{1}=\operatorname{Fr} U \subset \bigcup_{i=1}^{k} \operatorname{Fr} U_{i}$ is a partition between $A_{1}$ and $B_{1}$ and satisfies (5). For $i \geq 2$ there exists a partition $L_{i}^{\prime}$ between $A_{i} \cap L_{1}$ and $B_{i} \cap L_{1}$ in $L_{1}$ such that the set $\bigcap_{i=2}^{N} L_{i}^{\prime}$ is compact for some integer $N$. Now if we take partitions $L_{i}$ between $A_{i}$ and $B_{i}$ in $X$ such that $L_{i} \cap L_{1} \subset L_{i}^{\prime}$, then the $\operatorname{set} \bigcap_{i=1}^{N} L_{i}$ is compact.

Corollary. For a space $X$ defined in Example 6.1 the transfinite compactness degree is not defined and there is no embedding $h: X \rightarrow I^{\omega}$ such that the set $\overline{h(X)} \backslash h(X)$ is $\mu-S$-w.i.d. (since $X$ does not satisfy (5)).

\section{REFERENCES}

[1] J. M. Aarts and T. Nishiura, Kernels in dimension theory, Trans. Amer. Math. Soc., 178 (1978), 227-241.

[2] P. S. Aleksandrov and B. A. Pasynkov, Vvedenie v teoriju razmernosti, Moskva 1973. (Introduction to Dimension Theory.)

[3] R. Engelking, Dimension Theory, Warszawa 1978.

[4] , Transfinite dimension, in: Surveys in General Topology, edited by G. M. Reed, New York 1980, 131-161.

[5] J. de Groot and T. Nishiura, Inductive compactness as a generalization of semicompactness, Fund. Math., 58 (1966), 201-218.

[6] J. R. Isbell, Uniform spaces, Providence, R.I. 1964.

[7] K. Kuratowski, Topology, vol. II., New York 1968.

[8] L. Luxemburg, $O$ transfinitnych inductivnych razmernostjah, Dokl. Akad. Nauk SSSR 209 (1973), 295-298. (On transfinite inductive dimensions, Soviet Math. Dokl., 14 (1973), 388-393.) 
[9] _ On universal locally finite-dimensional spaces, General Topology and Appl., 10 (1979), 283-290.

[10] On compactifications of metric spaces with transfinite dimension, Pacific J. Math., 101 (1982), 399-450.

[11] E. Pol, Remark on compactifications of metrizable spaces, Topology and Appl., 15 (1983), 293-299.

[12] L. R. Rubin, R. M. Schori and J. J. Walsh, New Dimension-theory techniques for constructing infinite-dimensional examples, General Topology and Appl., 10 (1979), 93-102.

[13] E. G. Skljarenko, O razmernostnyh svoistvah beskonecnomernych prostranstv, Izv. Akad. Nauk SSSR, Ser. Math., 23 (1959), 197-212. (On dimensional properties of infinite dimensional spaces, Amer. Math. Soc. Trans1., Ser. 2, 21 (1962), 35-50.)

[14] M. Wójcicka, On separation properties of $K_{\omega}$, Coll. Math., (to appear).

Received October 21, 1983 and in revised form April 29, 1985.

UNIVERSITY OF WARSAW

Palac Kultury I NaUki, IXP

00-901 WaRszaWa, POLAND 


\section{PACIFIC JOURNAL OF MATHEMATICS EDITORS}

\author{
V. S. VARADARAJAN (Managing Editor) \\ University of California \\ Los Angeles, CA 90024 \\ Hebert Clemens \\ University of Utah \\ Salt Lake City, UT 84112 \\ Charles R. DePrima \\ California Institute of Technology \\ Pasadena, CA 91125
}

R. FINN

Stanford University

Stanford, CA 94305

HermanN FlaschKa

University of Arizona

Tucson, AZ 85721

RAMESH A. GANGOLlI

University of Washington

Seattle, WA 98195

ROBION KIRBY

University of California

Berkeley, CA 94720

ASSOCIATE EDITORS
R. ARENS
E. F. BECKENBACH
B. H. NEUMANN
F. WOLF
K. YoshidA (1906-1982)

C. C. Moore

University of California

Berkeley, CA 94720

H. SAMELSON

Stanford University

Stanford, CA 94305

HAROLD STARK

University of California, San Diego

La Jolla, CA 92093

\section{SUPPORTING INSTITUTIONS}

UNIVERSITY OF ARIZONA

UNIVERSITY OF BRITISH COLUMBIA

CALIFORNIA INSTITUTE OF TECHNOLOGY

UNIVERSITY OF CALIFORNIA

MONTANA STATE UNIVERSITY

UNIVERSITY OF NEVADA, RENO

NEW MEXICO STATE UNIVERSITY

OREGON STATE UNIVERSITY
UNIVERSITY OF OREGON

UNIVERSITY OF SOUTHERN CALIFORNIA

STANFORD UNIVERSITY

UNIVERSITY OF HAWAII

UNIVERSITY OF TOKYO

UNIVERSITY OF UTAH

WASHINGTON STATE UNIVERSITY

UNIVERSITY OF WASHINGTON 


\section{Pacific Journal of Mathematics}

Vol. 122, No. $1 \quad$ January, 1986

Michael James Cambern, Near isometries of Bochner $L^{1}$ and $L^{\infty}$ spaces ....1 Kun Soo Chang, Gerald William Johnson and David Lee Skoug, The

Feynman integral of quadratic potentials depending on two time

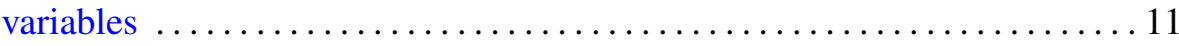

Robert Coleman, One-dimensional algebraic formal groups $\ldots \ldots \ldots \ldots \ldots 35$

Alberto Collino, The Abel-Jacobi isomorphism for the cubic fivefold .......43

N. J. Dev and S. S. Khare, Finite group action and vanishing of $N_{*}^{G}[F] \ldots 57$

Harold George Diamond and Jeffrey D. Vaaler, Estimates for partial sums

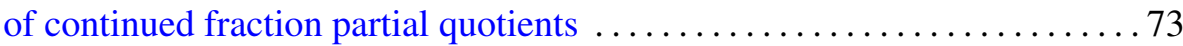

Kenneth R. Goodearl, Patch-continuity of normalized ranks of modules

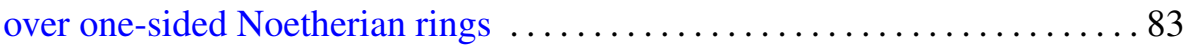

Dean Robert Hickerson and Sherman K. Stein, Abelian groups and

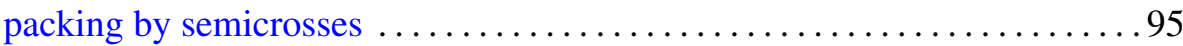

Karsten Johnsen and Harmut Laue, Fitting structures $\ldots \ldots \ldots \ldots \ldots 11$

Darren Long, Discs in compression bodies . ................... 129

Joseph B. Miles, On the growth of meromorphic functions with radially distributed zeros and poles ........................... 147

Walter Volodymyr Petryshyn, Solvability of various boundary value problems for the equation $x^{\prime \prime}=f\left(t, x, x^{\prime}, x^{\prime \prime}\right)-y \ldots \ldots \ldots \ldots \ldots . \ldots 169$

Elżbieta Pol, The Baire-category method in some compact extension problems

Masami Sakai, A new class of isocompact spaces and related results 211

Thomas Richard Shemanske, Representations of ternary quadratic forms and the class number of imaginary quadratic fields ..

Tsuyoshi Uehara, On class numbers of cyclic quartic fields 